EMBRYARIDDLE
Aeronautical University

SCHOLARLY COMMONS
International Journal of Aviation, Aeronautics, and Aerospace

3-7-2016

\title{
Peer Reviewed Safety Management System (SMS): Collaboration for Continuous Improvement (Literature Review)
}

\author{
Michael F. Canders Ph.D. CFII \\ Farmingdale State College, New York, canderm@farmingdale.edu
}

Follow this and additional works at: https://commons.erau.edu/ijaaa

Part of the Aviation Safety and Security Commons, and the Management and Operations Commons

\section{Scholarly Commons Citation \\ Canders, M. F. (2016). Peer Reviewed Safety Management System (SMS): Collaboration for Continuous Improvement (Literature Review). International Journal of Aviation, Aeronautics, and Aerospace, 3(2). https://doi.org/10.15394/ijaaa.2016.1113}

This Literature Review is brought to you for free and open access by the Journals at Scholarly Commons. It has been accepted for inclusion in International Journal of Aviation, Aeronautics, and Aerospace by an authorized administrator of Scholarly Commons. For more information, please contact commons@erau.edu. 
Aviation safety, from the rudimentary beginning of aviation to the technology-laden present day, has evolved greatly. Aviation grew rapidly in the United States following World War II, as commercial air travel became accessible to the general public. Aircraft accidents during this period of rapid growth were often attributed to technical factors associated with the introduction of larger airframes, jet engines, and swept back wings (Rodrigues 2012).

During the 1950s and 1960s, the main safety focus was mechanical improvements and better technology. This focus was further sharpened in the 1970s during the Total Quality Management (TQM) movement fostered by quality experts such as W. Edwards Deming and Joseph Juran. The result of this movement was the improved manufacturing and effective introduction of technology into finished products such as modern aircraft (Rodrigues 2012).

The discourse surrounding aviation safety shifted to human factors after many of the technical or material factors were ruled out as causes of aircraft accidents. Crew resource management (CRM) and human performance became important topics for pilot and aircrew training in the 1970s after a series of tragic aircraft accidents were attributed to human error (Rodrigues 2012). As airlines in the United States and around the world began to grow in organizational size and flourish between the 1970s and the 1990s, a different perspective about aviation safety began to emerge. Safe operations were viewed as a function of an interconnected system, comprised of internal organizational elements and external elements, such as regulations. Both internal and external factors had a significant overlay of human factors.

In the late 1990s, the International Civil Aviation Organization (ICAO), the United Nations global forum for aviation issues, began to develop a global aviation safety management plan (ICAO 2014). It published the first version of this plan in 1997. The ICAO Global Aviation Safety Plan (GASP) was updated regularly until 2005 when the ICAO began the transition to an integrated systems approach to aviation safety, which is outlined in its 2006 Safety Management Manual. This manual provided the guidance for establishment of Aviation SMS by the UN member states (ICAO 2014). 
The Federal Aviation Administration (FAA) adopted the provisions of this ICAO SMS approach to aviation safety in August of 2010 through its Advisory Circular number 120-92A (FAA 2010). The FAA describes SMS as a fundamental business practice that will integrate risk management and safety assurance into repeatable, proactive processes (FAA 2015). The FAA recommends that aviation service providers take an active role in accident prevention through an effective, but in many cases voluntary, SMS program that will provide:

- a structured means of risk management decisionmaking

- a means of demonstrating safety management capability before system failures occur

- and risk controls through structured safety assurance processes

- an effective interface for knowledge sharing between regulator and certificate holders

- a safety promotion framework to support a sound safety culture (FAA 2015)

While the FAA describes what aviation service providers should do in order to implement an effective SMS program, it does not specify how service providers should do this (FAA 2010a). There is an implied requirement for documentation, but exactly what that SMS document will look like is determined by the individual aviation service provider.

In the summer of 2009, the FAA Office of Aviation Safety (FAA AVS) issued an Advanced Notice of Proposed Rulemaking (ANPRM) to solicit public comments regarding the issuance of potential FAA regulations that would require virtually all aviation service providers to develop and implement SMS. This regulation would apply to certificate holders under US Federal Aviation Regulations, 14 CFR Parts 21, 119, $121,125,135,141,142$, and 145; it would also apply to product manufacturers, applicants, and employers (Federal Register 2009).

In October of 2010, the FAA moved forward to implement SMS for Part 139 certificated airports and for Part 121 certificate holders who are typically described as the major airlines in the United States. In March of 2011, the FAA withdrew the original 2009 ANPRM that proposed a much broader implementation of SMS, including withdrawal of the SMS requirement for a Part 141 training operation (Federal Register 2011). Part 
141 is frequently associated with the operational flight portion of collegiate aviation programs in the United States. Although aviation service providers, other than certificated airports and major airlines, are not required to implement SMS presently, the FAA recommends development of such programs on a voluntary basis.

Freiwald, Lenz-Anderson, and Baker (2013) assessed the aviation safety culture of a multinational flight training organization after two United States campuses of the organization experienced four fatalities in two separate aircraft accidents, and the loss of five aircraft in a very short 15-month period of time between April 2010 and July 2011. They used statistical analysis of a quantitative survey instrument and a qualitative interview of key leadership of the organization. Their key findings indicated that this organization did not have an effective safety culture, and trusted that the employees would behave in a safe manner only because they feared having an accident on their personal record that could ruin their career. The researchers strongly recommended implementation of a safety culture and SMS (Freiwald, Lenz-Anderson, Baker 2013).

\section{Collegiate Aviation Programs}

A significant number of colleges and universities in the United States and around the world offer aviation degrees. AvScholars, a website that cites itself as a student gateway to aviation, indicates that there are over 300 two- and four-year colleges with aviation programs in the USA and around the world (AvScholars 2015). The Aircraft Owners and Pilots Association (AOPA) lists 171 collegiate aviation programs in the United States (AOPA 2015). Many of these colleges and universities offer flight training as part of their curriculum, and operate a pilot school organized in accordance with 14 CFR Part 141. These collegiate pilot schools don't have a requirement for implementation of SMS because in 2011 the FAA withdrew the 2009 proposed SMS rulemaking for Part 141 operators and many other service providers. In their 2011 ANPRM withdrawal notice, however, the FAA did state that they may initiate additional rulemaking in the future to consider SMS for other product/service providers, but they did not provide a timetable for such implementation (Federal Register 2011).

Collegiate aviation programs have a special opportunity to influence future pilots, future aviation leaders, and future thought in this critical domain of aviation safety. Implementation of a SMS, while not required by the FAA, can provide a framework for the essential preparation necessary for tomorrow's aviation professionals to function 
effectively in an increasingly complex global aviation environment. Colleges or universities seeking the specialized accreditation offered by the Aviation Accreditation Board International (AABI) for their aviation programs must have and use a verifiable, formal aviation safety program that involves students, faculty, and staff for operations involving flight, maintenance, avionics and other aviation laboratories. The institution's aviation safety program must incorporate SMS key components appropriate to its national regulators guidance with that institution's size and scope, and should be coordinated with the institutions overall safety program (AABI 2013). As of 2015, the AABI website lists 33 different colleges and universities as having fulfilled this requirement to incorporate SMS key components in their safety programs however, this is only about $10 \%$ of the over 300 worldwide aviation programs cited by AvScholars (AvScholars 2015).

The format of the currently voluntary SMS program for Part 141 flight schools is not specified or constrained by the FAA or AABI, so it is the responsibility of the aviation service provider to choose a means that effectively communicates the principles and responsibilities associated with SMS programs. The FAA has provided a SMS section on their main website that may provide an excellent resource for those looking to implement a program. The website includes a framework document for voluntary implementation of SMS programs (FAA 2010). In July 2013, a team of researchers from the University of North Dakota issued a report with recommendations on how a Part 141 Pilot School could design and implement SMS that meets the requirements of the FAA SMS (Ullrich 2013). The FAA sponsored the research which resulted in the report. The work may provide a very useful tool kit for Part 141 Pilot School SMS implementation, and was completed by researchers from a US-based University with robust aviation program curricula.

The time may be right to promote the development of SMS by all collegiate aviation programs through publication of important peerreviewed SMS work.

\section{Review of Literature}

Published collegiate aviation program SMS may provide a ready library for collaboration in the college and university aviation community. Rather than simply publishing a college or university aviation program SMS, however, the work might be peer-reviewed by practitioners in the community, and perhaps published in a secured online environment 
accessible by password only to those who have made a submission. This password protected approach may seem exclusionary or elitist, but is proposed initially as an incentive for SMS authors to gain access to the specific SMS work of others by first making a tabula rasa SMS contribution without previous influence. After a trial period, the password protection might be removed in favor of other contribution incentives that may develop.

Peer review of scientific and technical work has long been established as a means to validate the writings of practitioners who sought to add value to society through knowledge. Publication of SMS work has a potential to add value and knowledge to the society of collegiate aviation programs. The Royal Society of London for Improving Natural Knowledge adopted a review procedure in 1752 for its journal, "Philosophical Transactions," in which publications in the journal were subject to inspection by members of the society who were knowledgeable in the documented matters (Spier 2002). Technology such as the typewriter and the copy machine would further facilitate the ability of reviewers to examine the works of others as the breadth and depth of scientific and technical publishing increased in the 19th and 20th centuries. The computer and internet technology of the 21 st century provides infinite opportunities for the publication of science and technical articles (Spier 2002).

Grainger (2007) describes peer review as a professional responsibility, and cites its value in validating scientific and technical publishing. He points out that peer review can ensure quality control only if the participants are willing to provide timely, unbiased, and ethical feedback to authors. SMS peer reviewers can be selected from the pool of collegiate aviation program safety experts, especially those who have authored a program and are actively implementing an SMS program at their college or university. There is a wealth of aviation safety experience in the ranks of collegiate aviation faculty and professional staff around the world. There is great benefit in being a peer reviewer, as it provides an opportunity to examine the safety management programs of others in seeking best practices to incorporate in one's own program.

Jennings (2006) writes that peer review provides great opportunity for increased quality and value. Authors who know that their work will be scrutinized by two or three others have an incentive to create a document that will represent the reputation they wish to establish or maintain as a practitioner. Jennings (2006) also points out that the process of revision 
after initial submission following the expert advice of a fellow practitioner can significantly improve the document, and benefit the author and future readers. Jennings (2006) also enumerates that the peerreviewed, post-submission revision process significantly improves documents to the benefit of the author and future readers.

Peer review is typically associated with scholarly journals, and many journals have migrated to the Internet via electronic online publishing. A collection of peer-reviewed SMS documents would probably not be published in a traditional hard copy journal, but (starting/having/maintaining) an electronic repository or library for SMS documents is an excellent solution. Schaffner (1994) discusses the role of scholarly journals for communities of interest in five functional ways:

- building a collective knowledge base

- communicating information

- validating the quality of the research

- distributing rewards

- building scientific communities

While the SMS library will not be a traditional hard copy journal, its existence as an online publishing repository will fulfill many of the roles described by Schaffner (1994). First and foremost, a collective knowledge base will be extremely valuable for continuous improvement of SMS programs. Contributors who successfully complete the peer review process will be granted access to the library and have the opportunity to review the work of others and seek best practices. This process reflects the second of Schaffner's functions for scholarly journals: communicating information. Sharing and communicating essential SMS information can have the effect of improving the SMS in use by other collegiate aviation programs, and therefore increase the general aviation safety margin. Those in the collegiate aviation SMS community can expect that the quality of the published SMS programs is validated through the review, revise, and continuous improvement approach. This represents the third function cited above.

Schaffner (1994) also discusses the rewards of publishing in a peerreviewed journal, and describes such publication as a way in which scholars are evaluated. Publishing a peer-reviewed SMS program should have substantial rewards for authors, since a high quality result can have enormous value through the safety dividends for flying operations. Finally, Schaffner's last function of building communities is an essential 
element of continuous improvement for all in the community. Connecting collegiate aviation programs in the safety domain and allowing constant discourse in an effective forum can build this community which has an enormous responsibility to prepare tomorrow's professional pilots for safe operation in an increasingly complex national and global airspace.

The many benefits of peer review are in contrast to a fair amount of criticism. Richard Smith, who was the editor of the British Medical Journal (BMJ) and chief executive of the BMJ Publishing Group for 13 years, cites a number of deficiencies (Smith 2006). First, he contends that it is slow and expensive, even with the speed and efficiency of the Internet. He cites opportunity cost, or the time spent peer reviewing, as time that could be spent on something else. He considers this a major expense. Slowness is a function of the ability of the reviewers to complete the task, because tablets, smart phones, and other modern communication electronics certainly allow for speed once the review is completed. Safety should be a preeminent consideration for collegiate aviation programs, so the incentive to review a quality SMS publication in a timely fashion should be natural. Reviewers who prioritize and complete tasks that have the most value avoid opportunity costs. Safety practitioners who volunteer to review SMS publications are choosing to spend their time in a way that add significant value to their practice.

Next, Smith (2006) cites inconsistency as a weakness of peer review, despite some reputation as a reliable, objective, and consistent process. He gave the following example of two reviewers commenting on the same paper:

- Reviewer A: "I found this paper an extremely muddled paper with a large number of deficits"

- Reviewer B: "It is written in a clear style and would be understood by any reader"

Smith provided no evidence that this extreme example occurs with any frequency. Different reviewers will most likely assess any publication differently, and that is actually a strength of the process.

Next, Smith (2006) discusses bias as a flaw in the peer review process. He cites evidence of bias against women authors and authors from institutions of low prestige. He also gave an example of pressure to accept a poor quality paper while he was the editor of the British Medical 
Journal (BMJ) from a well-known practitioner whose name recognition was very high in their BMJ community of scholars. A ready way to overcome this bias would be to remove the name and the institution of the author, but there can be occasions when this would be very difficult; especially in the relatively small community of collegiate aviation programs. Some of the bias may be due to the fact that scholarly journals have limited publication space, requiring competition among authors. An online library of SMS publications would have no such restrictions, and therefore reduce the risk of bias.

Smith's (2006) final criticism of peer review is the potential for abuse of the process. He describes stolen ideas or unjustly harsh or slow reviews to beat a competitor. Without the traditional competition for publication space described earlier, there may be less potential for this sort of abuse. Since the fundamental topic and subject matter of SMS publication will be the same for all authors, sharing ideas rather than stealing them will be the properly established framework.

\section{Peer review is viable for collegiate aviation programs SMS}

Despite receiving its share of criticism, peer review for SMS is feasible. Publications that are reviewed by experts in the aviation safety field who offer suggestions to the SMS authors for improvement can have the validity and quality that peer review provides. Smith's (2006) assertions that peer-reviewed work is slow and expensive, inconsistent, and subject to bias and abuse can be addressed with the establishment of an online SMS publication library. Publication in the library would require the endorsement of dedicated peer reviewers and achievement of standards that meet the requirements of the ICAO and FAA. Additionally, there would be no limit on the number of publications, which would reduce competition.

\section{A Model for Peer-Reviewed SMS}

Schaffner (1994) described building scientific communities as an important function for peer- reviewed scholarly journals. A peer-reviewed online library of important documents can also be a way to build a community of specific interest in areas such as SMS. There are a number of ways to build and maintain such a library.

Within the collegiate aviation domain, there are US-based organizations that provide a community of collegiate aviation interests in 
their membership. Among these organizations are the University Aviation Association (UAA) and the Aviation Accreditation Board International (AABI). The mission of the UAA is to promote and foster excellence in collegiate aviation education by providing a forum for students, faculty, staff, and practitioners to: share ideas, enhance the quality of education, develop stronger programs and curricula, influence aviation education policy at all governmental levels, and provide and nurture the linkage between college aviation education, the aviation industry, and government agencies (UAA 2015). An online password-protected publication library for SMS programs to foster continuous collaboration is strongly aligned with their mission statement, so such a library would seem natural for the UAA.

According to their mission statement, the AABI advances quality aviation education worldwide through accreditation and leadership (AABI 2015). As previously stated, the AABI does require accredited collegiate aviation programs to have and use a verifiable, formal aviation safety program that involves students, faculty and staff for operations involving flight, maintenance, avionics, and other aviation laboratories. The institution's aviation safety program must incorporate SMS key components appropriate to its national regulator's guidance regarding institution size and scope, and should be coordinated with the institution's overall safety program (AABI 2015). A peer-reviewed SMS program document would satisfy the verifiable portion of the safety program criteria for AABI accreditation. Both organizations actively promote the development of SMS programs through safety workshops offered at their annual and semi-annual conferences. However, the profile and importance of safety in collegiate aviation programs could be significantly elevated from the current level of promotion if peer-reviewed SMS programs are published and available for review by contributors who seek to continuously improve their systems. UAA or AABI seem to be logical places for the peer-reviewed SMS library to be built and maintained, and for management of the peer review and publication process. Other options might include a college or university that has a well-established reputation for its SMS and is interested in establishing a Part 141 SMS Center of Excellence (SMSCOE). They may already have the electronic infrastructure in place to build the SMS library. A good example of such an institution with these capabilities is the Embry-Riddle Aeronautical University (ERAU) Scholarly Commons.

Once the password-protected online library is established, the UAA or 
AABI could organize a team of peer reviewers with the expertise resident in college education programs. The process to fill the library with peerreviewed SMS publications could be as follows:

- SMS document(s) are submitted electronically by an author representing a collegiate aviation program

- The documents are reviewed by two or three peer reviewers for quality, validity, and compliance with FAA or ICAO SMS guidance

- Noncompliant documents are returned to the author with recommended changes

- Compliant, validated documents are published in an online, password-protected repository that is only accessible to contributors that have successfully completed the peer review process

- The SMS programs published in the online library are available for review by other contributors and practitioners who can reference a variety of approaches on designing and implementing an effective SMS

- Password protection might be removed or modified after a trial period of peer review to enable unconstrained sharing of best practices, but password protection seems to be an effective way to initially establish an incentive to contribute

- The library could also be constructed with an associated real-time, online blog or other means of commentary and communication between practitioners for questions and answers, and real-time discourse

- A committee of reviewers would have an opportunity to publish a best practices SMS in the library at some frequency; annually, for example 


\section{Conclusion}

An online, peer-reviewed SMS library with members-only access reserved for peer- reviewed contributors may have many benefits: it could build a community of specific interest; it could share vital safety knowledge; it could promote and improve safety in the general aviation domain; it may prompt other SMS research; it could foster creativity; it could encourage collaboration among practitioners and continuous improvement of individual programs; lastly, it could raise the profile and increase the prominence of aviation safety in collegiate programs. The quality of the library would be expected to improve with each submission as new ideas are brought forth and shared, and the library adds significant value to the society of collegiate aviation programs. Eventually, members-only password access may be considered for removal if other incentives develop for authors to contribute their work.

Successful implementation of the library may be of great interest to the ICAO or to the FAA. It could provide a model to encourage voluntary implementation of SMS programs in other sectors of the national or international aviation domain. This peer review process could be considered self-regulation with fewer requirements for oversight by regulators. The leaders of a successful and proven library could collaborate with the FAA or other international regulators to further promote and spread their SMS concepts.

There are other potential personal benefits to faculty or professional staff who author SMS. Scholarship, teaching, and service are traditional measures of performance for college and university professors, and are frequently used in making recognition, promotion, and term and continuing appointment decisions. Scholarship often equates to publishing scholarly works in peer-refereed journals. This scholarship should include publication of significant, relevant, and vitally important work such as an SMS.

College and university leaders of aviation programs have no greater responsibility than ensuring that their flying operations and aviation maintenance practices are safe. Collegiate aviation programs also have a special responsibility to prepare their graduates for safe and effective work in the global aviation industry through the provision of knowledge, preparation, and experience of operating within a wellestablished SMS. It is time to elevate the profile of SMS in the collegiate aviation program community by setting the bar higher through a peer- 
International Journal of Aviation, Aeronautics, and Aerospace, Vol. 3 [2016], Iss. 2, Art. 1

reviewed system that will facilitate collaboration for continuous improvement. 


\section{References}

Aircraft Owners and Pilots Association (2015). Flight colleges. Retrieved from

http://flighttraining.aopa.org/learntofly/school/aviation_colleges/co llegeresults.cfm

Aviation Accreditation Board International (2013). Accreditation criteria manual form 201. Auburn, Alabama: Author.

Aviation Accreditation Board International (2015). About AABI: Mission. Retrieved from http://www.aabi.aero/mission.html.

AvScholars (2015). Student gateway to aviation. Retrieved from http://www.avscholars.com/

Federal Aviation Administration FAA (2010). Advisory circular-AC 120 92A. SMS for aviation service providers. Washington DC: Author.

Federal Aviation Administration FAA (2010a). SMS (SMS) framework for SMS (SMS) pilot project participants and voluntary implementation of SMS programs. Washington DC: Author.

Federal Aviation Administration FAA (2015). SMS: SMS explained. Retrieved from http://www.faa.gov/about/initiatives/sms/explained/.

Federal Register (2009). SMS advance notice of proposed rulemaking. Retrieved from https://www.federalregister.gov/articles/2009/07/23/E917553/safety-management- system

Federal Register (2011). SMS; Withdrawal. Retrieved from https://www.federalregister.gov/articles/2011/03/17/2011$6255 /$ safety-management- system-withdrawal

Freiwald, D., Lenz-Anderson, C, \& Baker, E. (2013). Assessing safety culture within a flight training organization. Journal of Aviation/Aerospace Education and Research, 22(2). Retrieved from http://commons.erau.edu/db-applied - aviation/2. 
Grainger, D. W. (2007). Peer review as professional responsibility: A quality control system only as good as the participants.

Biomaterials, 28(2007), 5199-5203.

International Civil Aviation Organization (2014). The postal history of ICAO. Retrieved from http://www.icao.int/secretariat/PostalHistory/ annex_19_safety_management.htm

Jennings, C. J. (2006). Quality and value: the true purpose of peer review. Nature. doi: 10. 1038/nature 05032.

Rodrigues, C. C. \& Cusick, S. K. (2012). Commercial aviation safety (5 ${ }^{\text {th }}$ ed.). McGraw-Hill: New York.

Schaffner, A. C. (1994). The future of scientific journals: Lessons from the past. Information Technology and Libraries, 13, 239-247.

Smith, R. (2006). Peer review: a flawed process at the heart of science and journals. Journal of the Royal Society of Medicine, 99(April), 178182.

Spier, R. (2002). The history of the peer-review process. Trends in Biotechnology, 20(8), 357-358.

Ullrich, G.M. (2013). SMS (SMS) for FAA part 141 pilot schools. Washington DC: Federal Aviation Administration.

University Aviation Association (2015). About UAA: Our mission. Retrieved from http://www.imis100us1.com/UAA/UAAMain/About/Mission_Visi on_Objectives/UAAMa in/About_UAA/Copy_of_Mission Vision and_Objectives.aspx?hkey=1838f210-440c- 4368-9410$3 \mathrm{c} 8 \mathrm{c} 4 \mathrm{a} 236 \mathrm{c} 41$. 